Classification

Physics Abstracts

0.632

\title{
JAUGE DE PRESSION MINIATURE POUR BASSES TEMPÉRATURES
}

\author{
G. DELMAS et D. MARTY \\ Service de Physique du Solide et de Résonance Magnétique \\ Centre d'Etudes Nucléaires de Saclay, BP nº 2, 91190 Gif-sur-Yvette, France
}

(Reçu le 30 septembre 1974, accepté le 3 janvier 1975)

\begin{abstract}
Résumé. - Nous avons construit et étudié une jauge de pression à effet capacitif de petite dimension, conçue pour opérer entre 0 et 150 atm et à des températures inférieures à $4,2 \mathrm{~K}$.
\end{abstract}

Abstract. - We have built and studied a capacitive pressure gauge of small dimensions designed to measure pressures from 0 to $150 \mathrm{~atm}$, at temperatures below $4.2 \mathrm{~K}$.

La jauge de pression se présente comme une sonde miniature que l'on place dans une cellule à pression. Elle est constituée de deux pastilles plates métalliques moulées, sur leur pourtour, dans une résine synthétique (Stycast $2850 \mathrm{GT}$ ). Une variation de pression sur la jauge entraîne une variation de capacité du condensa-

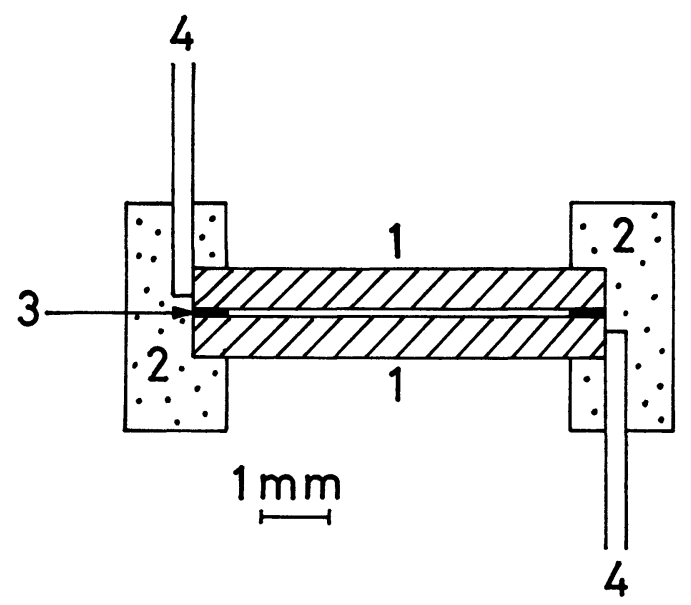

FIG. 1. - Coupe transversale de la jauge. 1) Pastilles en acier inoxydable. 2) Stycast. 3) Rondelle en mylar. 4) Fils conducteurs. teur formé par les deux pastilles. Ces pastilles (en acier inoxydable ou en cuivre-béryllium) ont une épaisseur de $0,6 \mathrm{~mm}$ et sont rectifiées et polies au micron près ; elles sont espacées et isolées électriquement par une rondelle de Mylar de $10 \mu$ d'épaisseur. (Ces chiffres peuvent être modifiés selon la gamme de pression que l'on désire étudier.) Chaque fil conducteur est soudé sur la tranche d'une pastille (soudure électrique ou soudure au fer suivant le matériau).

Les caractéristiques de cette jauge ont été étudiées avec un pont capacitif à trois entrées, qui permet d'obtenir six chiffres significatifs. La capacité varie de $21 \mathrm{pF}$ à $33 \mathrm{pF}$ quand la pression passe de 0 à $150 \mathrm{~atm}$. La sensibilité augmente avec la pression : elle est de $0,16 \mathrm{pF} / \mathrm{atm}$ à $150 \mathrm{~atm}$, ce qui correspond à une résolution de $5 \times 10^{-4} \mathrm{~atm}$, ou $3 \times 10^{-6}$ en valeur relative, en prenant $10^{-4} \mathrm{pF}$ comme résolution du pont capacitif. La reproductibilité, pour une pression donnée est meilleure que $10^{-4} \mathrm{pF}$, au cours d'un cycle de mesures (à peu près 4 h) sur 4 jours, la dérive est inférieure à $10^{-3} \mathrm{pF}$. Entre $1,25 \mathrm{~K}$ et $4,2 \mathrm{~K}$, nous n'avons pas décelé de variation de capacité pour différentes pressions (à la précision de $5 \times 10^{-3} \mathrm{pF}$ ), en fonction de la température. 